преподаватель кафедры бизнес-информатики и математического моделирования,

Крымский федеральный

университет им. В.И. Вернадского

\section{ПРИМЕНЕНИЕ ПОСЛЕДОВАТЕЛЬНОСТЕЙ ФИШБЕРНА В КОГНИТИВНЫХ ГИБРИДНЫХ СИСТЕМАХ ПОДДЕРЖКИ ПРИНЯТИЯ РЕШЕНИЙ [1]}

\begin{abstract}
Аннотация:
В статье рассмотрена роль в экономике гибридной системы поддержки принятия решений (СППР), включающей известные методы и модели. Дан обзор публикаций по данной теме. Проанализировано развитие когнитивной экономики в настоящее время. Обоснована целесообразность включения когнитивных моделей в СППР. Отмечена роль лица, принимающего решения (ЛПР), в моделировании ситуаций, требующих знаний о законах развития экономических событий. Для принятия управленческих решений, адекватно учитывающих и особенности сложившейся ситуации, и предпочтения ЛПР, в комплекс моделей рекомендовано включать методы с применением последовательностей Фишберна, позволяющие дать корректную оценку распределения априорных вероятностей и являющиеся удобным инструментарием для анализа различных экономических ситуаций в гибридных СППР. Приведены определения основных понятий, связанных с последовательностями Фишберна, а также общая структура простейшей когнитивной гибридной СППР.
\end{abstract}

Ключевые слова:

последовательность Фишберна, система поддержки принятия решений, когнитивный метод, гибридная СППР.

\section{Lecturer, Department of Business Informatics and Mathematical Modeling, Crimean Federal University \\ THE APPLICATION OF FISHBURNE SEQUENCES IN COGNITIVE HYBRID DECISION SUPPORT SYSTEMS [1]}

Summary:

In this paper, the role in the economy of a hybrid decision support system (DSS) is considered, including well-known methods and models. A review of publications on this topic is given. The development of cognitive economy at the present time is analyzed. The relevance to the inclusion of cognitive models in DSS is grounded. The role of the decision maker in modeling situations requiring knowledge of the laws of economic events is noted. To make managerial decisions that adequately take into account both the particulars of the current situation and the preferences of the decision maker, it is recommended to include methods using Fishburn sequences in the complex of models that make it possible to give a correct estimate of the distribution of a priori probabilities. The definitions of the basic concepts associated with Fishburn sequences are given. Fishburn sequences are proposed as a convenient tool for analyzing various economic situations in hybrid DSS. The general structure of the simplest cognitive hybrid DSS is presented.

Keywords: sequence of Fishburn, decision support system, cognitive approach, hybrid decision support system.

В настоящее время практика менеджмента разработала ряд разнообразных методов и моделей, ориентированных на поиск решения управленческих задач в условиях неопределенности. Существующие классификации этих методов и моделей позволяют привести процесс управления к применению набора типовых моделей, которые можно модифицировать под конкретную задачу управления. Следует выделить гибридные системы поддержки принятия решений (СППР), так как построение оптимальной модели для принятия решений чаще основывается на применении не одного метода, а различных методов и моделей в комплексе. В последнее время появляется значительное количество публикаций, посвященных гибридным СППР.

Исследование В.А. Артюхиной [2] посвящено гибридной СППР в задачах управления интегрированными производственными структурами. Автором разработана гибридная СППР, объединяющая лучшие качества модельно-ориентированных и знаниевых систем.

В работе С.Б. Титова [33] рассмотрены гибридные алгоритмы анализа и обработки данных в задачах поддержки принятия решений. Автором описан алгоритм кластеризации и упорядочивания для классификации объектов в сфере городской жилой недвижимости на основе гибридных технологий, реализующих комплексное применение различных алгоритмов и методов искусственного интеллекта, применяемых в рамках теории искусственных иммунных систем, теории нечетких множеств, теории генетических алгоритмов, теории искусственных нейронных сетей, теории мультимножеств. 
В статье А.Н. Полетайкина [44] рассматривается гибридная СППР для экономического процесса продвижения товара на региональный рынок, основанная на нечетком и имитационном моделировании.

Исследование А.Н. Аверкина, С.А. Ярушева, В.Ю. Павлова [5] посвящено изучению использования гибридной СППР, включающей нечеткие когнитивные карты, для прогнозирования динамических ситуаций.

В статье О.В. Рогозина [6] рассмотрен программный комплекс анализа характеристик инновационного проекта, разработанный с помощью созданной нейро-нечеткой модели, благодаря которой можно получить эфрфективное решение для слабоструктурируемых задач на основе анализа качественных характеристик.

Основная задача всех СППР - обработка, анализ данных и изучение на их основе возможных альтернатив для принятия решения. Следует учитывать, что сам процесс получения взвешенных стратегических решений становится следствием объединения результирующего показателя после обработки и анализа данных с ощущением настоящего и нового течений в экономических процессах. Поэтому методология моделирования должна учитывать знания экспертов о законах развития моделируемой ситуации. В экономике при принятии решений в условиях неопределенности и риска особая роль отводится лицу, принимающему решение (ЛПР). Ведь именно от выбора ЛПР зависит исход. В свою очередь данное лицо опирается на имеющуюся информацию, свои собственные знания в рассматриваемой области и опыт, полученный за определенный промежуток времени. Анализ ситуации, выбор альтернатив и принятие решения ЛПР являются предметом исследования когнитивной экономики.

На сегодняшний момент имеется достаточно большое количество работ, посвященных различным направлениям развития когнитивной экономики, как зарубежных, так и отечественных исследователей. Например, Н.М. Абдикеевым, А.Н. Аверкиным, Н.А. Ефремовой [7] когнитивная экономика рассматривается в широком и узком смыслах. С точки зрения данных авторов, в широком смысле она состоит из интеллектуальных систем, управления знаниями и когнитивной экономики в узком смысле. Пересечение данных областей порождает такие направления современной экономики, как бизнес-аналитика, гибридные интеллектуальные системы, когнитивный маркетинг. Методы и модели, применяемые в данных направлениях, охватывают весь процесс принятия решения ЛПР, начиная от анализа и оценки сложившейся ситуации и заканчивая выбором оптимальной альтернативы.

Для принятия управленческих решений, адекватно учитывающих и особенности сложившейся ситуации, и предпочтения ЛПР, в комплекс моделей рекомендовано включать методы с применением последовательностей Фишберна [8]. Данные методы позволяют разрабатывать модели, использующие когнитивные методологии. Например, в статической ситуации применяются методы поддержки принятия решений на основе теории выбора. При решении статистической игры важно корректно оценить распределение априорных вероятностей состояний «природы», т. е. экономической среды. Для этого целесообразно применять последовательности Фишберна (см., например, [9, с. 132]).

Приведем определения основных понятий, связанных с последовательностями Фишберна.

Пусть $\left\{a_{j}\right\}_{j=1}^{n}-$ произвольная монотонная последовательность неотрицательных чисел, сумма которых является положительным числом, т. е. справедливы соотношения $a_{1} \geq a_{2} \geq \ldots \geq$ $\geq a_{j} \geq \ldots \geq a_{n} \geq 0$ или $0 \leq a_{1} \leq a_{2} \leq \ldots \leq a_{j} \leq \ldots \leq a_{n}$, при этом $\sum_{j=1}^{n} a_{j}>0$, тогда последовательность $\left\{q_{j}\right\}_{j=1}^{n}$,

где $\quad q_{j}=\frac{a_{j}}{\sum_{i=1}^{n} a_{i}}, j=\overline{1, n}$, удовлетворяет следующим свойствам:

1) условию нормировки $\sum_{j=1}^{n} q_{j}=1$;

2) требованиям неотрицательности всех элементов $q_{j} \geq 0, j=\overline{1, n}$;

3) простому линейному отношению порядка [10, с. 78], согласно которому последовательность $\left\{q_{j}\right\}_{j=1}^{n}$ должна обладать свойством монотонности.

Последовательностью Фишберна будем называть последовательность $\left\{q_{j}\right\}_{j=1}^{n}$, значения элементов которой вычисляются по формулам (1), где $\left\{a_{j}\right\}_{j=1}^{n}-$ монотонная последовательность неотрицательных чисел, сумма которых является положительным числом, при этом последовательность $\left\{a_{j}\right\}_{j=1}^{n}$ представляется последовательностью, производящей (или порождающей) последовательность Фишберна $\left\{q_{j}\right\}_{j=1}^{n}$. Очевидно, что любая последовательность Фишберна может задавать распределение вероятностей, например, распределение вероятностей состояний экономической среды.

Последовательности Фишберна представляют собой удобный инструментарий, позволяющий выполнять соответствующий анализ различных экономических ситуаций и процессов, а также 
принимать управленческие решения в экономике. Применение последовательностей Фишберна в нечетком когнитивном моделировании, в моделях с количественными фракторами, а также в теоретико-игровых моделях подробно рассмотрено в других наших работах, в частности, в монографии [11], которая также включает анализ конкретных экономических ситуаций и рекомендации по построению весовых коэффициентов. Интеграция методов, основанных на использовании последовательностей Фишберна, в разработанные модели СППР позволяет адекватно и корректно учитывать важные особенности современной экономики (неопределенность, неполноту информации, конфликтность, многокритериальность и порожденный ими экономический риск), специфику сложившейся ситуации принятия управленческих решений, а также предпочтения ЛПР.

Для принятия взвешенного стратегического решения СППР должна включать современные цифровые модели анализа данных с обязательным учетом знаний о возможном развитии экономической ситуации. Объединение данных моделей можно назвать когнитивными гибридными СППР. Например, простейшая когнитивная гибридная СППР - это комплекс моделей, включающих обработку и анализ данных совместно с конечной оценкой полученных альтернатив для принятия решений. ЛПР задает вектор весовых коэффициентов для определенного метода оценки. Так, общую структуру простейшей когнитивной гибридной СППР можно представить в следующем виде (рис. 1).

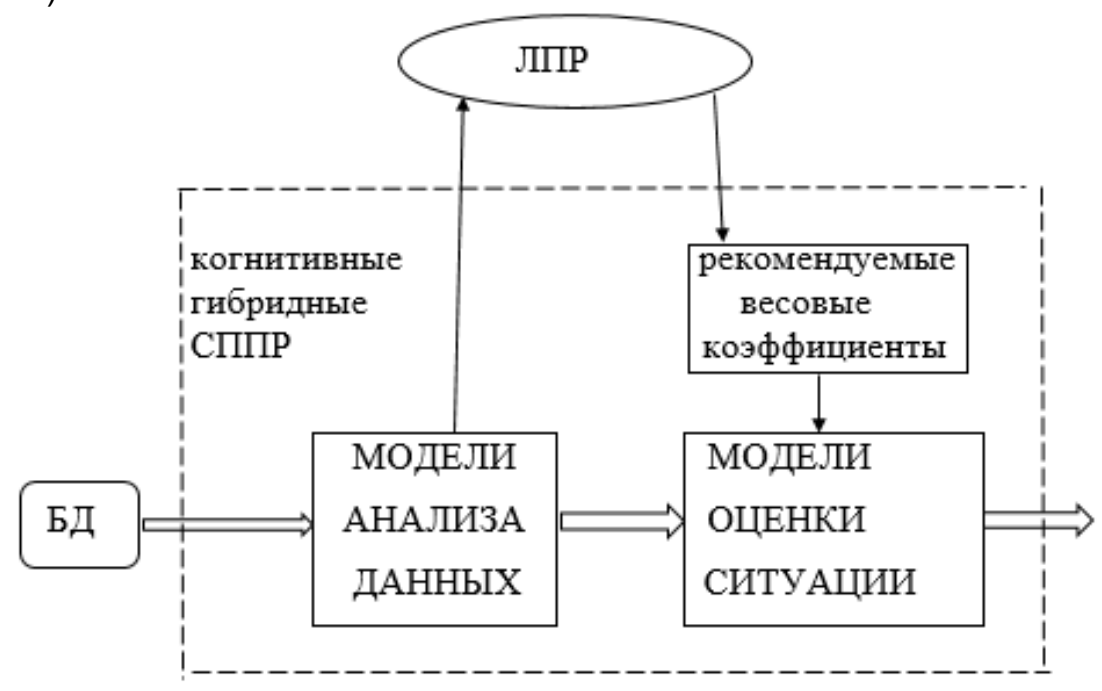

Рис. 1 - Общая структура СППР с применением когнитивного подхода

Для более качественного анализа сложившейся ситуации и выбора дальнейшего действия необходимо создавать автоматизированные СППР, адаптированные под конкретное предприятие на основе когнитивных методов.

При принятии управленческих решений следует отметить решающее значение опыта, компетентности и профессиональной интуиции ЛПР. Это относится не только к выбору применяемых методов и моделей, но и к реализуемым в моделях оценкам - например, оценкам распределения вероятностей возможных состояний экономической среды или оценкам вектора весовых коэффрициентов. Для выбора этих оценок, адекватно учитывающих и особенности сложившейся ситуации, и предпочтения ЛПР, целесообразно применять последовательности Фишберна, обладающие желаемыми свойствами.

\section{Ссылки и примечания:}

1. Исследование выполнено при финансовой поддержке РФФИ в рамках научного проекта № 18-010-00688.

2. Артюхина В.А. Гибридная система поддержки принятия решений в задачах управления интегрированными производственными структурами: автореф. дис. ... канд. техн. наук. Уфа, 2013. 16 с.

3. Титов С.Б. Гибридные алгоритмы анализа и обработки данных в задачах поддержки принятия решений: автореф. дис. ... канд. техн. наук. Рязань, 2011. 17 с.

4. Полетайкин А.Н. Гибридный подход к построению системы поддержки принятия решений при продвижении товаров на региональный рынок // Вестник СибГУТИ. 2015. № 1. С. 45-59.

5. Аверкин А.Н. Когнитивные гибридные системы поддержки принятия решений и прогнозирования / А.Н. Аверкин, С.А. Ярушев, В.Ю. Павлов // Программные продукты и системы. 2017. № 4 (30). С. 632-642. https://doi.org/10.15827/0236235x.120.632-642.

6. Рогозин О.В. Оценка инновационной привлекательности проекта с использованием нейро-нечеткого адаптивного программного комплекса // Инженерный журнал: наука и инновации. 2012. № 1 (1). С. 26-38. http://dx.doi.org/10.18698/23086033-2012-1-13. 
7. Абдикеев Н.М. Когнитивная экономика в эпоху инноваций / Н.М. Абдикеев, А.Н. Аверкин, Н.А. Ефремова // Вестник РЭА. 2010. № 1 (31). С. 3-20.

8. Ремесник Е.С. Последовательности Фишберна и их применение в экономических исследованиях / Е.С. Ремесник, А.В. Сигал. Симферополь, 2019. 188 с.

9. Сигал А.В. Последовательности Фишберна и их применение в современной теории портфеля / А.В. Сигал, Е.С. Ремесник. Симферополь, 2018. 204 с.

10. Там же.

11. Ремесник Е.С. Указ. соч.

Редактор: Ситникова Ольга Валериевна Переводчик: Бирюкова Полина Сергеевна 